\title{
Meningiomas induced by low-dose radiation carry structural variants of NF2 and a distinct mutational signature
}

\author{
Felix Sahm ${ }^{1,2}$ • Umut H. Toprak ${ }^{3}$ - Daniel Hübschmann ${ }^{3,4,5}$ • Kortine Kleinheinz ${ }^{3}$ - Ivo Buchhalter ${ }^{3}$. \\ Martin Sill $^{6} \cdot$ Damian Stichel $^{2} \cdot$ Matthias Schick $^{7} \cdot$ Melanie Bewerunge-Hudler $^{7}$ - Daniel Schrimpf ${ }^{1,2}$. \\ Gelareh Zadeh $^{8,9} \cdot$ Ken Aldape $^{8,10}$ • Christel Herold-Mende ${ }^{11}$ - Katja Beck ${ }^{12}$ - Ori Staszewski ${ }^{13}$ - Marco Prinz ${ }^{13,14}$. \\ Carmit Ben Harosh ${ }^{15}$ - Roland Eils ${ }^{3,4,12} \cdot$ Dominik Sturm $^{5,16}$ • David T. W. Jones ${ }^{16}$ • Stefan M. Pfister ${ }^{5,16}$. \\ Werner Paulus $^{17} \cdot$ Zvi Ram $^{15,18} \cdot$ Matthias Schlesner $^{3} \cdot$ Rachel Grossman $^{15,18} \cdot$ Andreas von Deimling $^{1,2}$
}

Meningiomas not only occur sporadically or in the context of neurofibromatosis type 2 , but can also be induced by radiation [7]. High-dose radiation-induced meningiomas (Mrad) predominantly arise after radiation for other brain tumors (often in childhood) with doses of 40-60 Gy. Most low-dose Mrad have been detected in patients receiving 3-4 Gy to the scalp for tinea capitis [6, 9]. Analysis of these tumors is paradigmatic for radiation-induced

Felix Sahm, Umut H. Toprak, Daniel Hübschmann, Matthias Schlesner, Rachel Grossman, and Andreas von Deimling: shared first/senior authors.

Felix Sahm

felix.sahm@med.uni-heidelberg.de

Andreas von Deimling

andreas.vondeimling@med.uni-heidelberg.de

1 Department of Neuropathology, Institute of Pathology, University Hospital Heidelberg, Heidelberg, Germany

2 Clinical Cooperation Unit Neuropathology, German Consortium for Translational Cancer Research (DKTK), German Cancer Research Center (DKFZ), Heidelberg, Germany

3 Division of Theoretical Bioinformatics (B080), German Cancer Research Center (DKFZ), Heidelberg, Germany

4 Department for Bioinformatics and Functional Genomics, Institute for Pharmacy and Molecular Biotechnology (IPMB) and BioQuant, Heidelberg University, Heidelberg, Germany tumorigenesis, particularly in the absence of a prior tumor and chemotherapy and a model for collateral exposure surrounding the target of high-dose irradiation. So far, little molecular overlap between Mrad and sporadic meningiomas has been detected $[6,9]$.

We here performed whole genome sequencing of 20 meningiomas induced by low-dose radiation (L-Mrad). Age, gender, and WHO grade are given in the supplementary tables. However, the documentation lacked the date of irradiation and the exact histological subtype, which could also not be determined retrospectively due to freezing artifacts. Whole genome sequencing of these 20 L-Mrad with matched germline controls revealed single nucleotide variations (SNVs) of NF2 in 6/20 (30\%) cases, in line with $25 \%$ detected by Sanger sequencing in a previous L-Mrad cohort [6]. 2/20 (10\%) cases had small insertions/deletions (InDels) within NF2 (SNVs and InDels in Supplementary Tables). The advantage of genome sequencing is the ability to identify structural

5 Department of Pediatric Immunology, Hematology and Oncology, University Hospital Heidelberg, Heidelberg, Germany

6 Division of Biostatistics, German Cancer Research Center (DKFZ), Heidelberg, Germany

7 Genomics and Proteomics Core Facility, Microarray Unit, German Cancer Research Center (DKFZ), Heidelberg, Germany

8 McFeeters-Hamilton Centre for Neuro-oncology, Princess Margaret Cancer Centre, Toronto, Canada

9 Division of Neurosurgery, Department of Surgery, University of Toronto, Toronto, Canada

10 Department of Laboratory Medicine and Pathobiology, University of Toronto, Toronto, Canada 
variants (SVs): We detected two cases $(10 \%)$ with truncating NF2 gene rearrangements (example depicted in Fig. 1a, SVs for all cases in Supplementary Figure 1).

Collectively, 10/20 (50\%) L-Mrad exhibited genomic variants affecting the $N F 2$ gene, indicating that NF2 inactivation is a key mechanism in L-Mrad, similar to sporadic meningioma. Other genes associated with sporadic meningioma [2-4] were not affected.

All other SNVs and InDels affected individual genes in one or two L-Mrad only. In addition, we analyzed SV data for regional enrichments of aberrations (Fig. 1b): 18/20 (90\%) patients had a chromosome (chr) $1 \mathrm{p}$ loss, $6 / 20$ (30\%) cases had a loss of chr7p, and 5/20 (25\%) cases had rearrangements on chr19p13;12-13. These findings were confirmed by copy number plots derived from methylation data. Comparison to sporadic meningioma revealed that the $7 p$ deletions occur exclusively in L-Mrad, most frequently in cases with grade I histology (Fig. 1c, Supplementary Figure 2). For comparison, $450 \mathrm{k}$ data of a cohort of seven Mrad induced by high-dose radiation were available. These high-dose Mrad had a low frequency of $7 p$ deletions (Supplementary Figure 2). Collectively, a high number of SVs were common in the L-Mrad cohort.

Next, we performed a supervised analysis for the presence of the mutational signatures introduced by Alexandrov et al. [1,5] (Supplementary Figure 3a), here referred to as $A C$ for "Alexandrov COSMIC". Several mutational signatures have been reported so far and some are specific for physiological or pathological mechanisms, e.g., spontaneous deamination, or exogenic mutagens, e.g. tobacco smoke. L-Mrad exhibited a rather homogeneous spectrum of mutational signatures (Fig. 1d): We detected signature AC1 (known to result from spontaneous

11 Division of Experimental Neurosurgery, Department of Neurosurgery, University Hospital Heidelberg, Heidelberg, Germany

12 Heidelberg Center for Personalised Oncology (DKFZ-HIPO), German Cancer Research Center (DKFZ), Heidelberg, Germany

13 Institute of Neuropathology, Faculty of Medicine, University of Freiburg, Freiburg, Germany

14 BIOSS Centre for Biological Signalling Studies, University of Freiburg, Freiburg, Germany

15 Department of Neurosurgery, Tel-Aviv Medical Center, Tel-Aviv, Israel

16 Division of Pediatric Neurooncology, German Consortium for Translational Cancer Research (DKTK), German Cancer Research Center (DKFZ), Heidelberg, Germany

17 Institute of Neuropathology, University Hospital Muenster, Muenster, Germany

18 Sackler School of Medicine, Tel-Aviv University, Tel-Aviv, Israel deamination), AC3 (associated with defects in DNA double-strand break repair by homologous recombination), AC5 (mechanism unknown), and AC25 (mechanism unknown). Although AC3 is associated with homologous recombination repair (HRR) deficiency, only one L-Mrad carried a deleterious mutation and another harboured an SNV of unknown significance in an HRR gene. Moreover, an analysis of mutational signatures for their location in the genome showed enrichment of the mutational signature AC3 in the vicinity of breakpoints (Fig. 1e). This indicates a relation of these SNVs with genomic rearrangements and suggests that they might be a consequence of genomic rearrangements caused by irradiation. Intriguingly, the only infratentorial meningioma (Mrad_8) in our cohort (Supplementary Figure 4) did not show the mutational signature AC3. Since this case also has an SNV in NF2 and virtually no SVs, it might actually represent an NF2 mutant sporadic meningioma.

Next, SNVs were stratified for cellular fractions (CFs). CFs are calculated from the mutant allele frequency, corrected for tumor cell content, tumor ploidy, and allelespecific copy-number. This allows separation of SNVs arising early in clonal evolution from later events. Direct comparison between CFs and proximity to breakpoints revealed that the early SNVs are enriched near breakpoints (Fisher exact test $p=0.01182$ ). Intriguingly, these SNVs near breakpoints showed predominantly mutational signature AC3 (Fig. 1f, Supplementary Figure 3b). These findings strongly support our hypothesis that AC3 SNVs are the fingerprint of early, radiation-induced genomic rearrangements.

Finally, we asked if sporadic and radiation-induced meningioma originate from the same cell of origin. Epigenetic profiling is able to group tumors according to their origin population and is able to distinguish clinically relevant subgroups of meningioma [8]. Unsupervised clustering of $450 \mathrm{k}$ methylation data from 40 sporadic meningiomas with $N F 2$ or $A K T 1$ mutations and $31 \mathrm{~L}-$ Mrad revealed no individual group for L-Mrad (Supplementary Figure 5). Instead, they clustered predominantly with the NF2mutated sporadic meningiomas. Further studies are needed to identify whether the predominant localization of L-Mrad at the convexity is only due to the axis of radiation. Given the epigenetic and mutational similarity of sporadic meningioma of the convexity and L-Mrad, the findings might potentially also point toward differential susceptibility for radiation-induced tumor formation, particularly by $N F 2$ mutations, in arachnoidal cells of the convexity compared to those of the skull base.

In conclusion, we demonstrate a high frequency of SVs, including novel truncating fusions of $N F 2$, and an HRRindependent evolution of AC3 signature in low-dose radiation-induced Mrad. 
a

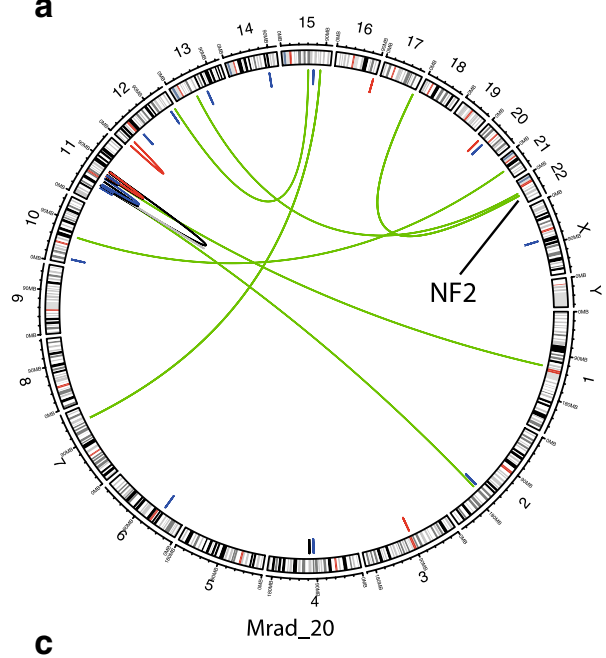

b

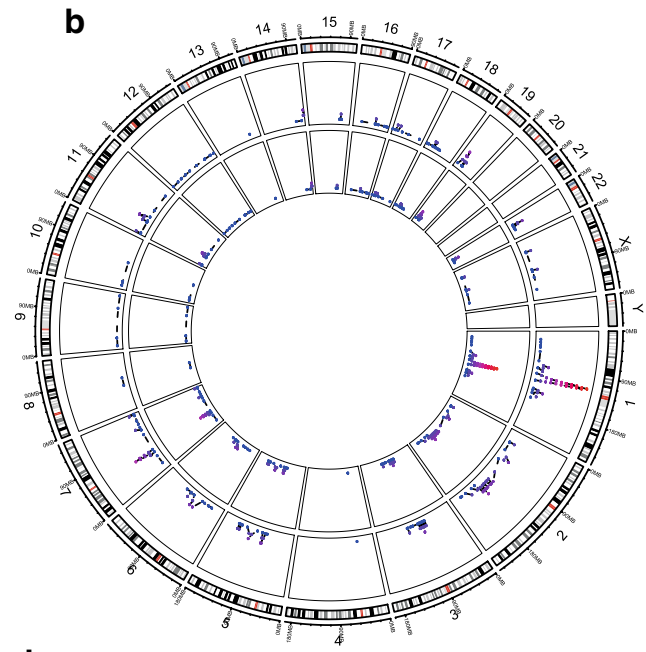

d

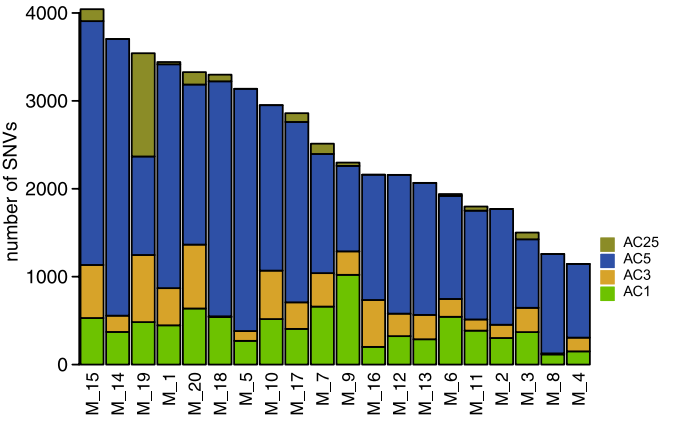

f

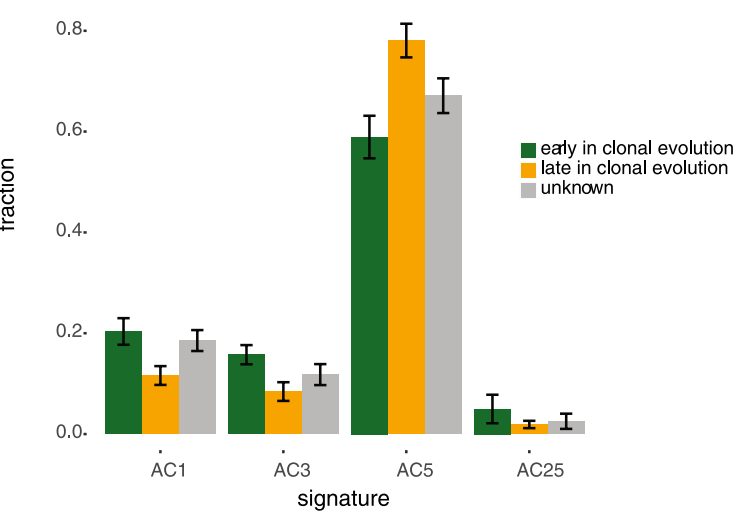

Fig. 1 a Circos Plot for Mrad_20 with SV involving NF2. b Cohortwide recurrent structural variant loci for the L-Mrad cohort. Each dot represents one genomic region in which at least two patients have SVs with a $2 \mathrm{MB}$ flanking region touching the peaks, normalized to 12 (of 20), the highest peak. c Relative copy number aberrations of 17 L-Mrad with WHO grade I histology. d Absolute number of mutational signatures in the L-Mrad cohort. The y-axis shows the absolute number of SNVs, and the height of the stacked barplots corresponds to the number of SNVs of the respective signature. e Relation between SNVs and SV breakpoints. Analysis of mutational signatures stratified by breakpoint proximity. SNVs categorized by their dis- tance to the closest SV breakpoint: less than $100 \mathrm{kBp}$, between 100 $k B p$ and $1 \mathrm{MBp}$ and more than $1 \mathrm{MBp}$. AC3 is enriched in the vicinity of breakpoints. $\mathbf{f}$ Analysis of mutational signatures stratified by cellular fraction (CF). Analogue to e, cohort-wide SNVs were categorized into variants arising early in clonal evolution of the tumor (corresponding to high values of $\mathrm{CF}$, i.e., $\mathrm{CF} \geq 0.5$ ) and variants arising late in the clonal evolution of the tumor (corresponding to low values of $\mathrm{CF}$, i.e. $\mathrm{CF}<0.5$ ). Variants for which the $\mathrm{CF}$ could not be computed are classified as unknown. Signature AC3 is enriched in early and depleted in late clonal evolution 
Acknowledgements We thank H-Y Nguyen, L Dörner, J Baron, K Lindenberg, U Lass, and J Meyer for skillful technical assistance and the Genome and Proteome Core Facility, High-Throughput Sequencing Unit of the DKFZ. This work was supported by the German Cancer Aid (110670, 110983), the Else Kröner-Fresenius Foundation (A_60), the DKFZ-HIPO through H033, the Hartmut HoffmannBerling International Graduate School (DH), the MD/PhD-program of the University Heidelberg (DH), and the Helmholtz International Graduate School for Cancer Research (UHT).

\section{References}

1. Alexandrov LB, Nik-Zainal S, Wedge DC et al (2013) Signatures of mutational processes in human cancer. Nature 500:415-421

2. Brastianos PK, Horowitz PM, Santagata S et al (2013) Genomic sequencing of meningiomas identifies oncogenic SMO and AKT1 mutations. Nat Genet 45:285-289

3. Clark VE, Erson-Omay EZ, Serin A et al (2013) Genomic analysis of non-NF2 meningiomas reveals mutations in TRAF7, KLF4, AKT1, and SMO. Science 339:1077-1080
4. Clark VE, Harmanci AS, Bai H et al (2016) Recurrent somatic mutations in POLR2A define a distinct subset of meningiomas. Nat Genet 48:1253-1259

5. Huebschmann D, Gu Z, Schlesner M (2016) YAPSA: Yet Another Package for Signature Analysis. R package version 0.99 .10

6. Joachim T, Ram Z, Rappaport ZH et al (2001) Comparative analysis of the NF2, TP53, PTEN, KRAS, NRAS and HRAS genes in sporadic and radiation-induced human meningiomas. Int $\mathrm{J}$ Cancer 94:218-221

7. Louis DN, Perry A, Reifenberger G et al (2016) The 2016 World Health Organization Classification of Tumors of the Central Nervous System: a summary. Acta Neuropathol 131:803-820

8. Sahm F, Schrimpf D, Stichel D et al (2017) DNA methylationbased classification and grading system for meningioma: a multicentre, retrospective analysis. Lancet Oncol. doi:10.1016/ S1470-2045(17)30155-9

9. Shoshan Y, Chernova O, Juen SS et al (2000) Radiation-induced meningioma: a distinct molecular genetic pattern? J Neuropathol Exp Neurol 59:614-620 